\title{
Adaptive Neuro-Fuzzy Inference System Controller for Vibration Control of Reduced-order Finite Element Model of Rotor-Bearing-Support System
}

\author{
Abdur Rosyid ${ }^{1}$, Mohanad Alata ${ }^{1}$, Mohamed ElMadany ${ }^{2}$ \\ ${ }^{1}$ Department of Mechanical Engineering College of Engineering, \\ King Saud University Riyadh, Saudi Arabia \\ ${ }^{2}$ National Commission for Academic Assessment and Accreditation \\ Riyadh, Saudi Arabia \\ E-mail address: malata@ksu.edu.sa,mmadany@ncaaa.org.sa
}

\begin{abstract}
Keywords: adaptive neuro-fuzzy inference system (ANFIS), linear quadratic regulator (LQR), rotor-bearing-support system, vibration control
\end{abstract}

ABSTRACT. This paper evaluates the use of adaptive neuro-fuzzy inference system (ANFIS) controller to suppress the vibration in a rotor-bearing-support system, and compare the performance to LQR controller. ANFIS combines the smooth interpolation of fuzzy inference system (FIS) and the learning capability of adaptive neural network. The ANFIS controller design starts with initialization which includes loading the training data and generating the initial FIS. In this case, the gain values obtained from the LQR controller design previously conducted were used as training data for the ANFIS controller. After the training data is provided, the ANFIS controller learns through a certain optimization algorithm to adjust the parameters. In the current work, hybrid algorithm was used due to its faster convergence. To evaluate the performance, the ANFIS output was compared to the training data. From the evaluation, it can be concluded that ANFIS controller can replace LQR controller with no need to solve the LQR's Riccati equation. However, in the initialization process, it needs training data obtained from LQR control design. Furthermore, ANFIS controller can replace more than one LQR controllers with different weighting matrices $\mathrm{Q}$ and/or R. In a more general tone, ANFIS controller can serve as an effective controller, given any arbitrary speed-gain pairs as its training data. Finally, ANFIS controller can serve as a better controller than LQR as long as tuning can be conducted adequately for that purpose.

\section{INTRODUCTION}

Many studies have proposed intelligent control, including fuzzy control, neural network, and combination between both of them, to suppress vibration in nonrotating structures. In rotordynamic field, intelligent control has also been applied to suppress the vibration. For that purpose, many works have proposed the use of magnetic bearing as actuator. Filbo et al. [1] proposed fuzzy logic controller for active control of rotor vibration using active magnetic bearing (AMB) as actuator. Nagi et al. [2] applied fuzzy bang-bang relay control in a singleaxis AMB system. Chen et al. [3] applied a self-tuning fuzzy PID-type controller design for unbalance compensation in an active magnetic bearing. Tung et al. [4] proposed design of modelbased unbalance compensator with fuzzy gain tuning mechanism for an active magnetic bearing system. Couzon and Hagopian [5] investigated neuro-fuzzy active control of rotor suspended on AMB.

Beyond the intelligent control of rotordynamic system with magnetic bearings, Gong and Cao [6] proposed fuzzy proportional-integral-derivative control of an overhang rotor with double discs on active tilting pad journal bearings. Koroishi et al. [7] investigated the use of fuzzy control of rotor system by using electromagnetic actuator. Borges et al. [8] investigated 
the use of fuzzy controller for rotor-bearing vibration control by using smart actuators. Li et al. [9] proposed the use of self-learning control for vibration control of a rotating spindle using piezopusher bearings. Lim et al. [10] applied artificial intelligent feedback control to suppress vibration of high speed rotor using electrorheological fluid damper. Tammi et al. [11] proposed a novel adaptive repetitive algorithm for vibration control of a variable-speed rotor. Furtherly, Tammi [12] proposed gradient-based repetitive learning control for rotor vibration control. Buttini and Nicoletti [13] investigated self-identification algorithm for autonomous control of lateral vibration in flexible rotors.

This paper presents the use of adaptive neuro-fuzzy inference system (ANFIS) controller to suppress the vibration in a rotor-bearing-support system, and compare the performance to LQR controller. Rosyid [14] has previously evaluated the use of LQR controller to suppress the vibration of a rotor-bearing-support system.

Numerical example used in Ref [14], as shown in Figure 1, is used in this paper so that the comparison of the performance can be conveniently conducted. The example consists of a rotor, two bearings, and damped, flexible structure which support the bearings. The rotor consists of a shaft and several disks, and modeled by using Timoshenko finite element beam. The bearings are modeled by using some linear springs and dampers as shown in Figure 2. The springs and dampers represent the stiffness dan damping provided by the fluid film in the bearings, respectively. Moreover, the springs and dampers oriented in $\mathrm{x}$ and $\mathrm{y}$ direction represent the direct stiffness and damping, whereas the springs and dampers inclined between $\mathrm{x}$ and $\mathrm{y}$ direction represent cross-coupling stiffness and damping. Eventually, the bearing support is isotropic with negligible damping and cross-coupling stiffness. Prior to the control design, the model of the rotor-bearing-support system was reduced to obtain smaller degrees of freedom.

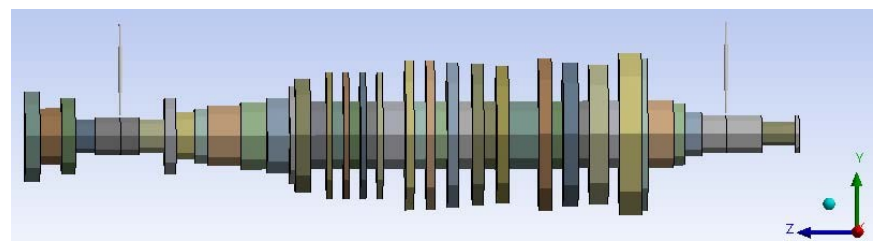

Figure 1. Finite element model of the rotor-bearing-support system used as numerical example

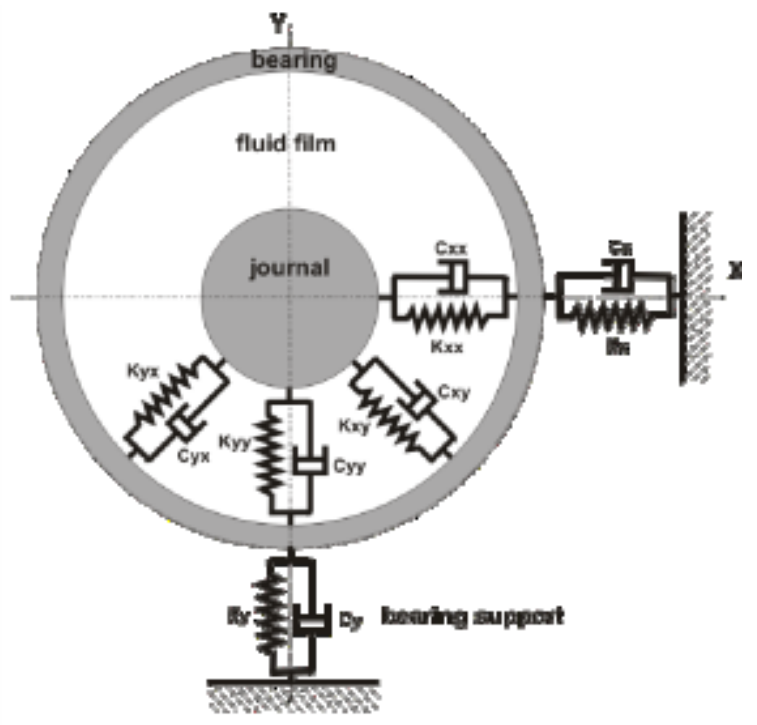

Figure 2. Model of the bearing mounted on damped flexible support

ANFIS, first introduced by Jang $[15,16]$, is adaptive network which serves functionally equivalent to Sugeno-type (and also Tsukamoto-type) fuzzy inference system. This system combines the advantages of both the adaptive network and the fuzzy inference system (FIS). The main advantage of neural network is its learning capability (sometimes called adaptability). On 
the other hand, fuzzy system offers smoothness but cannot learn. Applying FIS on a neural network frame, ANFIS has smoothness and learning capability at the same time. Given an input-output data, fuzzy rules (in this case: rules RHS of the Sugeno-type FIS) are constructed by itself through the learning process, and the FIS will interpolate among the rules to give smooth (fuzzy) output.

Learning algorithm in ANFIS may use one of the following four methods:

1) Only using back propagation.

2) Using back propagation and one pass least square estimates (LSE)

3) Using hybrid learning which combines back propagation and LSE

4) Using sequential LSE only

In MATLAB, the first and the third methods are available as options. The default option is the third method (i.e. the hybrid learning). It is to notice that LSE is computationally more complex than back propagation. The hybrid learning, as previously mentioned, converges faster compared to only back propagation.

In the hybrid learning, the set of total parameters consists of non-linear premise parameters and linear consequent parameters. Each epoch in the learning process is composed of forward pass and backward pass. The forward pass propagates the input vector through the network layer by layer. In other words, the node outputs are calculated layer by layer, until layer 4. In this pass, the non-linear premise parameters are fixed while the linear consequent parameters are computed using LSE. In the backward pass, the error is sent back through the network. In this pass, the linear consequent parameters are fixed while the nonlinear premise parameters are computed using back propagation. Table 1 summarizes these learning procedures.

TABLE 1. TWO PASSES IN THE HYBRID LEARNING OF ANFIS

\begin{tabular}{|l|c|c|}
\hline & $\begin{array}{c}\text { Forward } \\
\text { pass }\end{array}$ & Backward pass \\
\hline $\begin{array}{l}\text { Non-linear premise } \\
\text { parameters }\end{array}$ & Fixed & Back propagation \\
\hline $\begin{array}{l}\text { Linear consequent } \\
\text { parameters }\end{array}$ & LSE & Fixed \\
\hline \multicolumn{1}{|c|}{ Signals } & $\begin{array}{l}\text { Node } \\
\text { outputs }\end{array}$ & Error signals \\
\hline
\end{tabular}

In this work, MATLAB is used to conduct the ANFIS controller design and evaluate its performance. ANFIS in MATLAB only supports Sugeno-type FIS. Furthermore, it must have the following properties:

1) Be zero or first order Sugeno-type system.

2) Have a single output, obtained using weighted average defuzzification. All output membership functions must be the same type and be either linear or constant.

3) Have no rule sharing. Different rules cannot share the same output membership function, namely the number of output membership functions must be equal to the number of rules.

4) Have unity weight for each rule.

Moreover, the membership functions as well as the defuzzification functions cannot be customized. 


\section{PROCEDURES OF ANFIS CONTROLLER DESIGN}

In the current work, the ANFIS controller was built through the following steps, as described in the flowchart shown in Figure 3. In the initialization stage, firstly the training data is loaded. After that, FIS structure is generated either using genfis1 or genfis 2 commands. The genfis 1 command generates FIS structure from data using grid partition, whereas genfis 2 command generates FIS structure from data using subtractive clustering. Both the grid partition and the clustering are conducted to come up with less but representative number of rules. The grid partition is usually used to partition input space with a small number of MFs for each input. Moreover, it is not s uitable for moderately large number of inputs. On the other hand, the substractive clustering is an automatic data clustering which is usually used for quite large number of MFs for each input and/or quite large number of inputs, so that it will generate a FIS which best models the data behavior using a minimum number of rules. In the problem at hand, there is only one input and the number of MFs used is only 5 . This means there are only $5^{1}=5$ rules in the fuzzy system. For this reason, genfis1 is used instead of genfis2.

In the learning stage, anfis command is executed. This command runs the training process which stops whenever the designated epoch number is reached or the error tolerance is achieved. The error tolerance by default is set to 0. In the evaluation stage, the ANFIS output is plotted altogether (in the same axis) with the training data so that a comparison between them can be conducted. Finally, in the use stage, the FIS structure is written and saved. Prior to the assignment of the FIS structure (which contains gain values at different speeds) in the fuzzy logic controllers, the written FIS structure is read. Each fuzzy logic controller represents a gain element. All of the fuzzy logic controllers are then combined using muxes to function as a single controller subsystem with 2 x 60 gain elements as shown in Figure 4 . The controller subsystem is finally put in the whole control system.

The ANFIS control design was implemented to a rotor rotating on journal bearings supported by isotropic flexible structure. Two scenarios were used to evaluate the performance of the ANFIS control design. In the first scenario, the training data of the ANFIS controller was obtained from a single LQR controller. The objective of this scenario is that the ANFIS controller functions well as approximator of the LQR controller. In the second scenario, the gain elements of the ANFIS controller at lower speeds were trained from those of the LQR controller which gives better response at lower speeds (LQR 1). Accordingly, the gain elements of the ANFIS controller at higher speeds were trained from those of the other LQR controller (with different weighting matrices Q and R) which gives better response at higher speeds (LQR 2). The objective of this scenario is that the ANFIS controller combines the best response of both the LQR controllers. Furthermore, the ANFIS controller was further tuned in order to give better response in both the aforementioned scenarios. Table 2 shows brief information about the ANFIS control design conducted in the current work. 


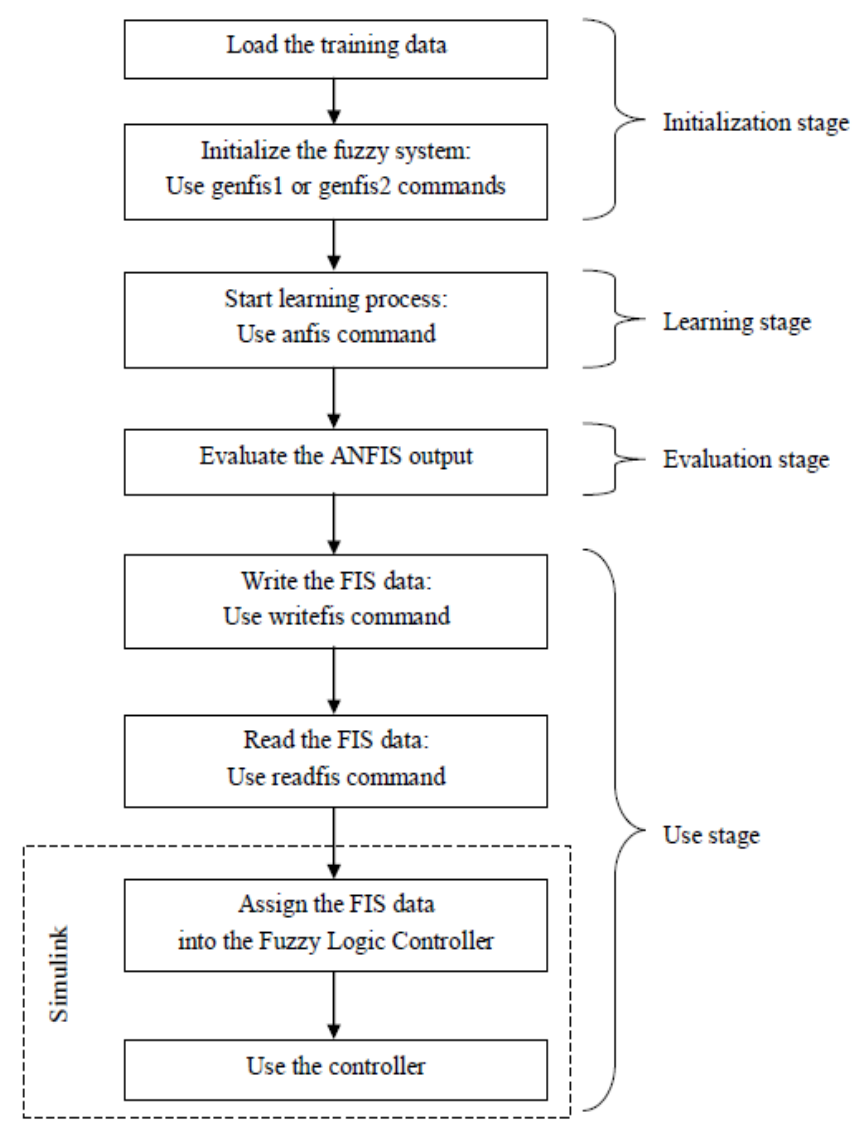

Figure 3. Finite element model of the rotor-bearing-support system used as numerical example

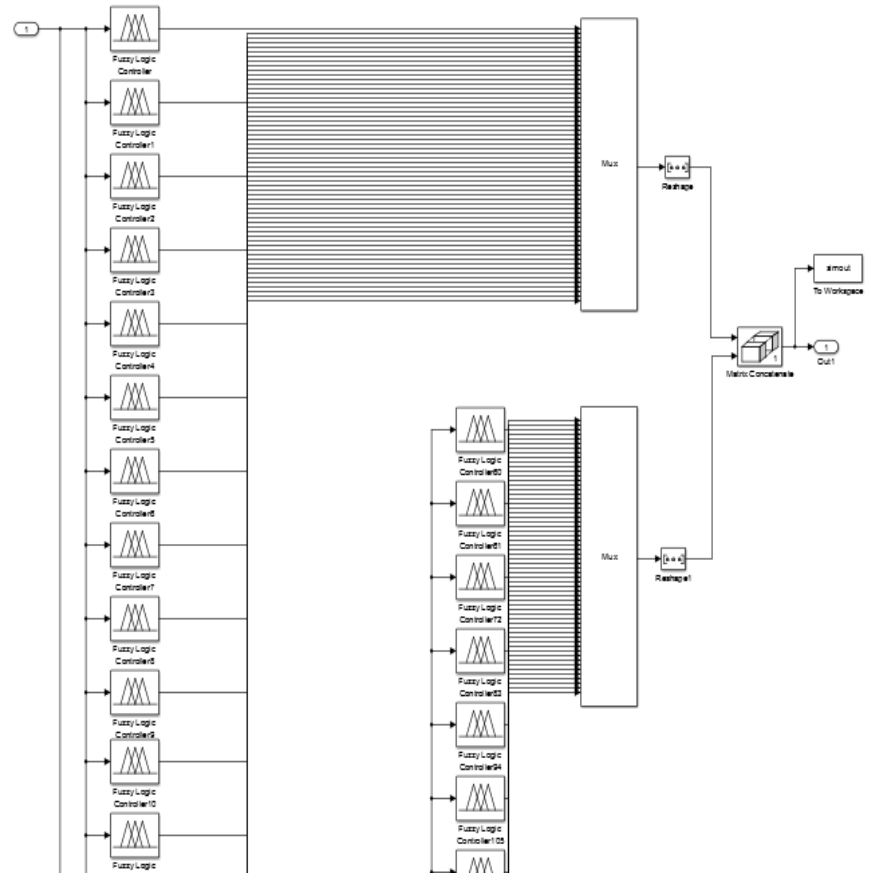

Figure 4. The gain elements combined using muxes to function as a single controller subsystem (some parts of the gain elements are not shown) 


\section{TABLE 2. TWO PASSES IN THE HYBRID LEARNING OF ANFIS}

\begin{tabular}{|l|l|}
\hline \multicolumn{1}{|c|}{ Parameter } & \multicolumn{1}{|c|}{ Value } \\
\hline Number of input & 1 (i.e. speed) \\
\hline Input MF & Mostly 'trimf', some are 'gbellmf' \\
\hline Number of output & 1 (i.e. gain element) \\
\hline Output type & Linear (first-order Sugeno type) \\
\hline Number of fuzzy rules & 5 \\
\hline $\begin{array}{l}\text { Number of input-output pairs } \\
\text { (training data) }\end{array}$ & \begin{tabular}{l} 
element) \\
\hline Learning algorithm pairs of speed - gain
\end{tabular} \\
\hline Number of nodes & Hybrid \\
\hline $\begin{array}{l}\text { Number of non-linear premise } \\
\text { parameters }\end{array}$ & 15 \\
\hline $\begin{array}{l}\text { Number of linear consequent } \\
\text { parameters }\end{array}$ & 10 \\
\hline Number of total parameters & 25 \\
\hline Number of epochs & 2000 \\
\hline Error tolerance & 0 \\
\hline Evaluation method & Training data versus ANFIS output \\
\hline
\end{tabular}

\section{ANFIS CONTROLLER AS APPROXIMATOR OF LQR CONTROLLER}

In this first scenario, the number of epochs was set to 2000 for the training process. For evaluation of the training, the result of ANFIS training was compared to the training data as shown in Figure 5. It is shown that for k160 (gain element at row 1 column 60), ANFIS gives a perfect approximation of the training data as both the curves are precisely coincident. The closedloop response of the system using ANFIS controller is shown in Figure 6. It is shown from the response that the ANFIS controller functions perfectly as approximator of the LQR controller. All of the responses using ANFIS controller are precisely coincident with those using LQR controller. The coincidence causes only a single controlled curve appears in the plots.

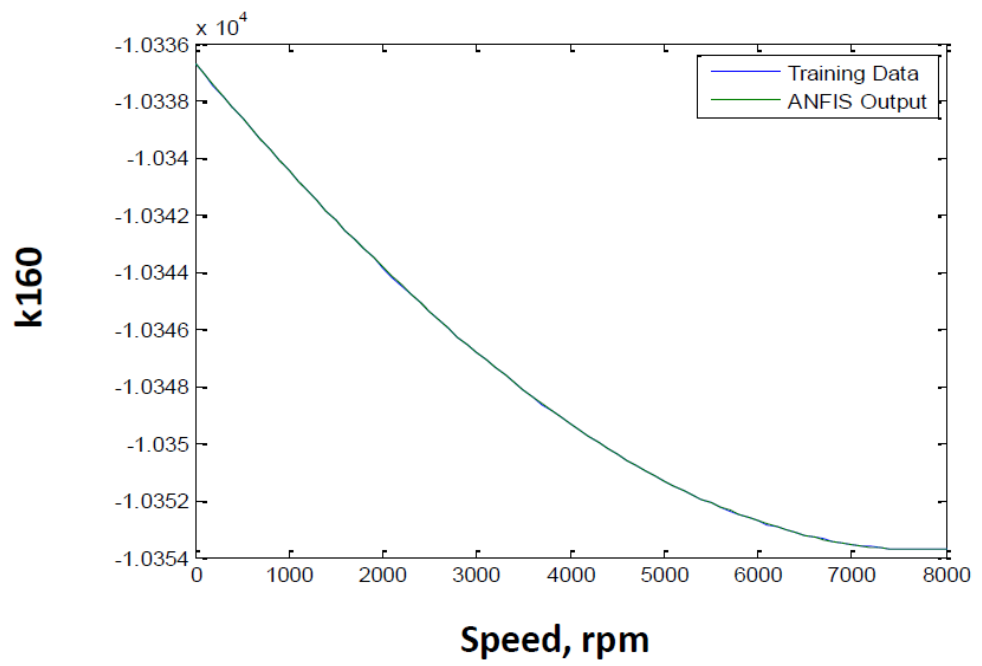

Figure 5. Comparison of the value of gain element k160 between the training data and the ANFIS training output in the first scenario 


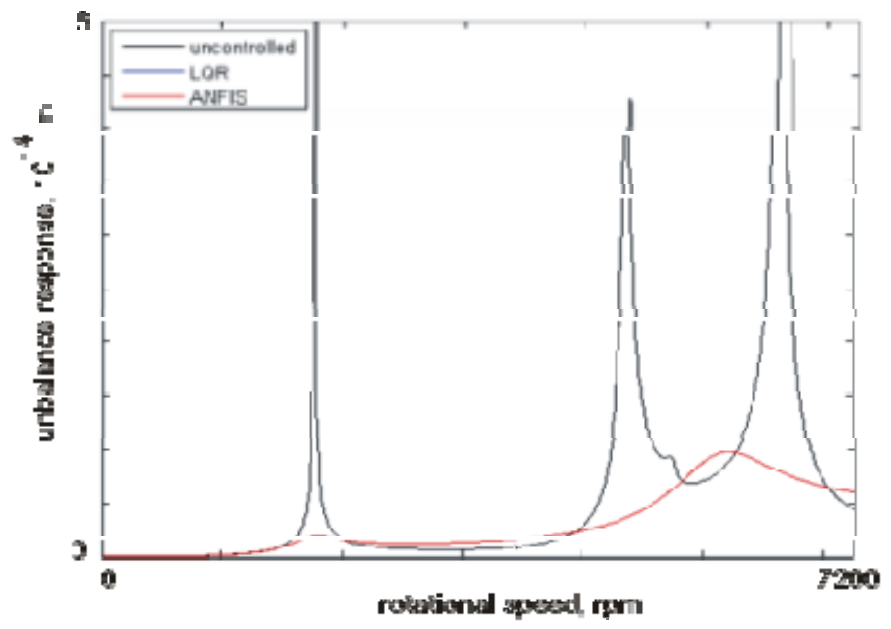

Figure 6. Response of the closed-loop system using ANFIS controller compared to that of the system using LQR controller in the first scenario, evaluated at the largest disk

\section{ANFIS CONTROLLER AS A SUBSTITUTE OF TWO DIFFERENT LQR CONTROLLERS}

As presented in Ref [14], two choices of weighting matrices were used in the LQR controller design. The first choice of the weighting matrices needs lower control force and gives quite good response in lower speed range. On the other hand, the second choice of weighting matrices gives better response particularly in higher speed range but needs larger control force. In this case, either the first or the second choice of weighting matrices is applied in all speed range $(0-8000$ RPM), or the first choice of weighting matrices are applied in lower speed range (02400 RPM) whereas the second choice of weighting matrices are applied in higher speed range (2400-8000 RPM). The latter option combines the advantages of both the first and the second choices and at the same time omitting the disadvantages of both. In lower speed range, lower control force is required and good response is given. Only when the rotor is rotating in higher speeds, larger control force is required to obtain better response.

In the latter option, weighting matrices change at the transition of lower and higher speeds (2400 RPM). Due to the change of the weighting matrices, the amount of control force also changes at the transition. Unfortunately, the change is abrupt. To overcome this abrupt change, a modification was conducted to give gradual transition between the two different weighting matrices. For k160, results of this modification are shown in Figure 7.

In this second scenario, the number of epochs was set at 2000. For evaluation of the training, the result of ANFIS training was compared to the training data as shown in Figure 8. It is shown that ANFIS gives a perfect approximation of the training data as both the curves are precisely coincident. Consequently, the ANFIS controller accomplishes the task quite well as shown in Figure 9. At lower speeds, the response of the system using the ANFIS controller coincides quite well with that of the system using LQR 1. Similarly, at higher speeds, the response of the system using the ANFIS controller coincides very well with that of the system using LQR 2. 


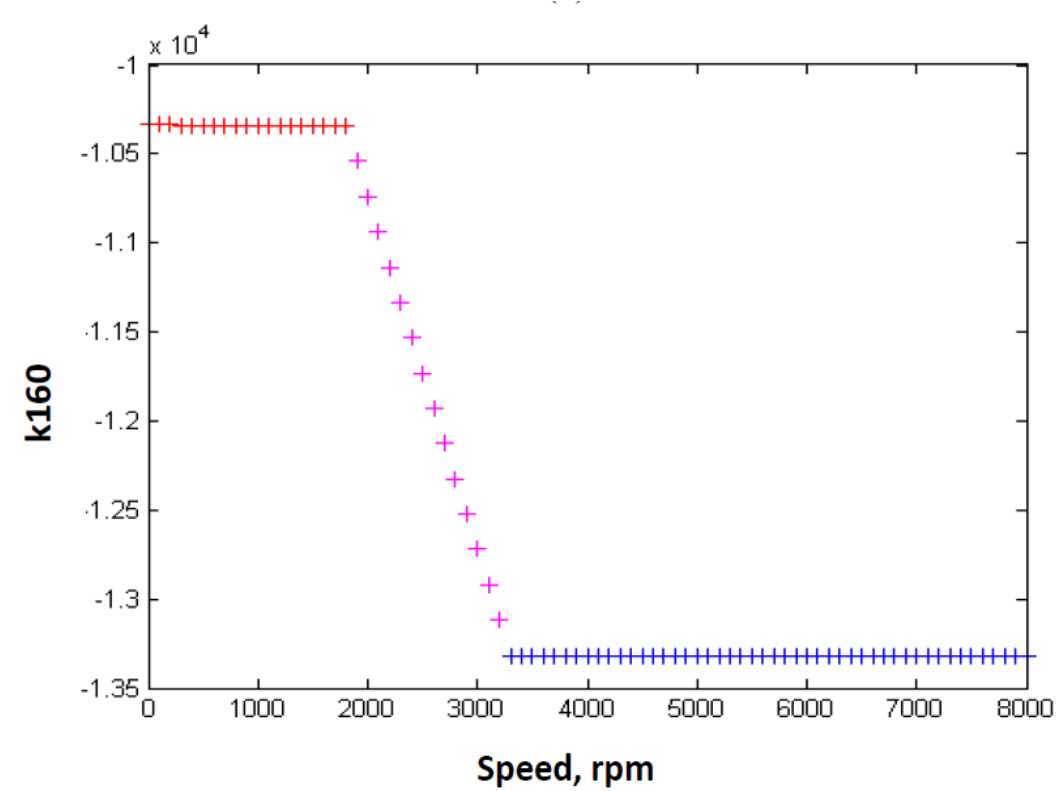

Figure 7. The values of gain element k160 along the speeds by using scenario 2 with modification at transition speeds

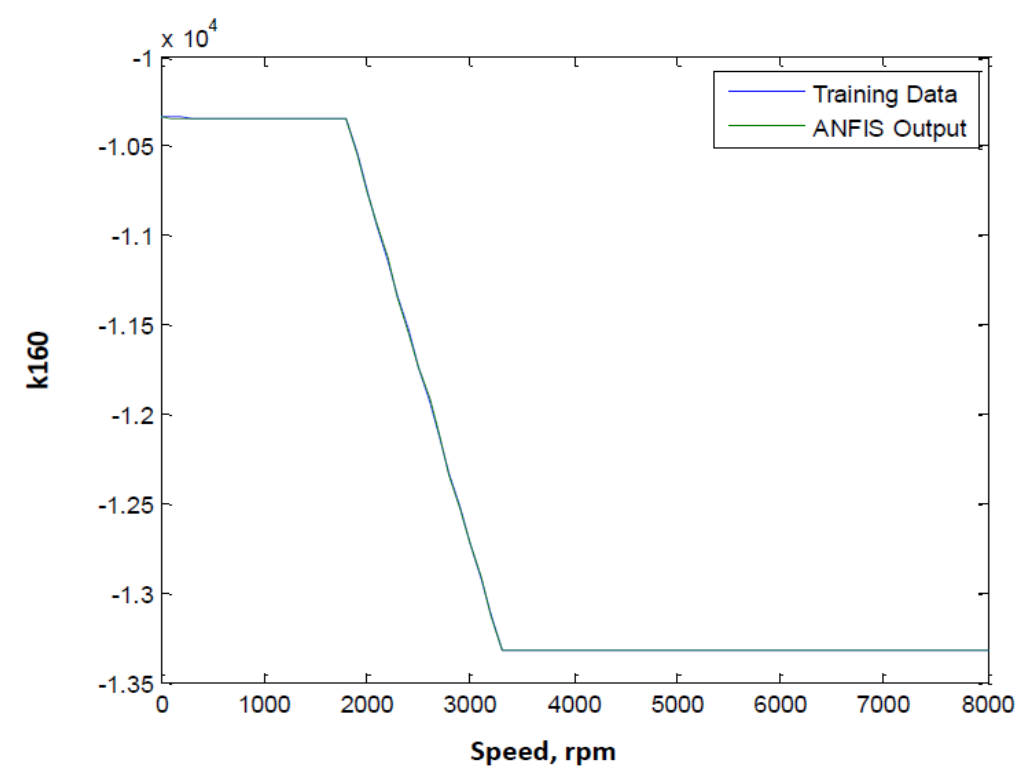

Figure 8. Comparison of the value of gain element k160 between the training data and the ANFIS training output in the second scenario 


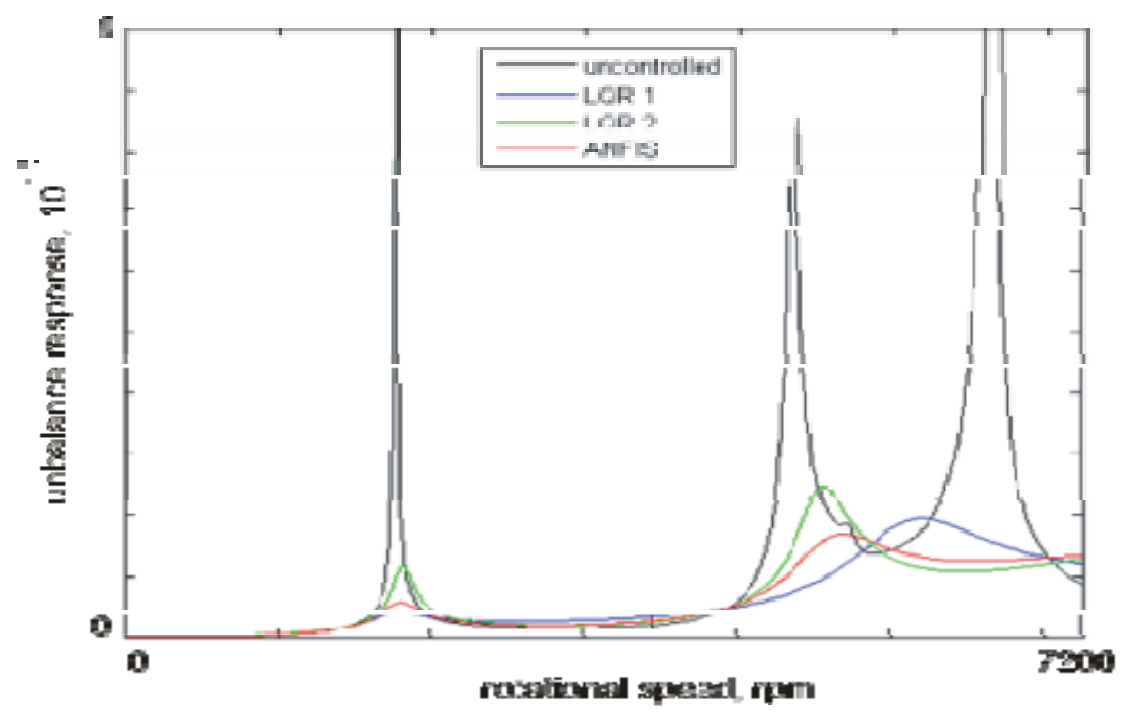

Figure 9. Response of the closed-loop system using ANFIS controller compared to that of the system using LQR controllers in the second scenario, evaluated at the largest disk

\section{TUNING OF ANFIS CONTROLLER TO OBTAIN BETTER PERFORMANCE}

In order to obtain better response, the ANFIS controller was tuned. This task was conducted in the two scenarios mentioned earlier. After some tuning trials, it was found that tuning scheme by putting constant gains before and/or after the whole ANFIS controller subsystem does not improve the response. Finally, the tuning was applied by adding constant gains before and/or after the gain elements inside the controller. All of the tuning schemes were done based on intuition, experience, and trial-and-error. It was shown that the latest tuning scheme gives better response to some extent. The response at higher speeds is better. Only very small oscilation occurs near the first critical speeds.

Using the first scenario, it is shown in Figure 10 that tuning the ANFIS controller gives better response. The tuning is conducted inside the ANFIS controller subsystem. Similarly to the tuning task in the first scenario, it is shown in Figure 11 that in the second scenario, tuning the ANFIS controller gives better response to some extent. The tuning is also conducted inside the ANFIS controller subsystem.

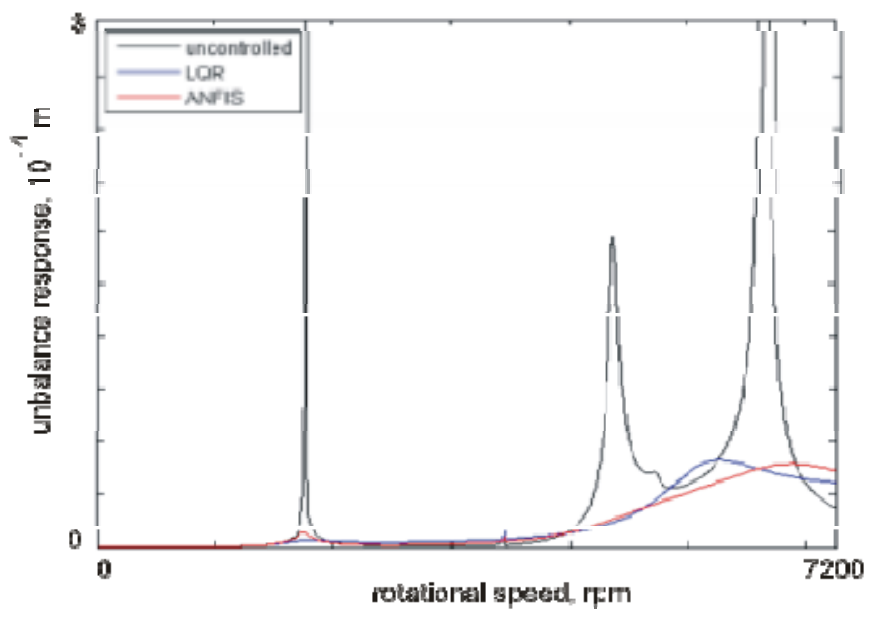

Figure 10. Response of the closed-loop system using tuned ANFIS controller compared to that of the system using LQR controller in the first scenario, evaluated at LPE bearing 


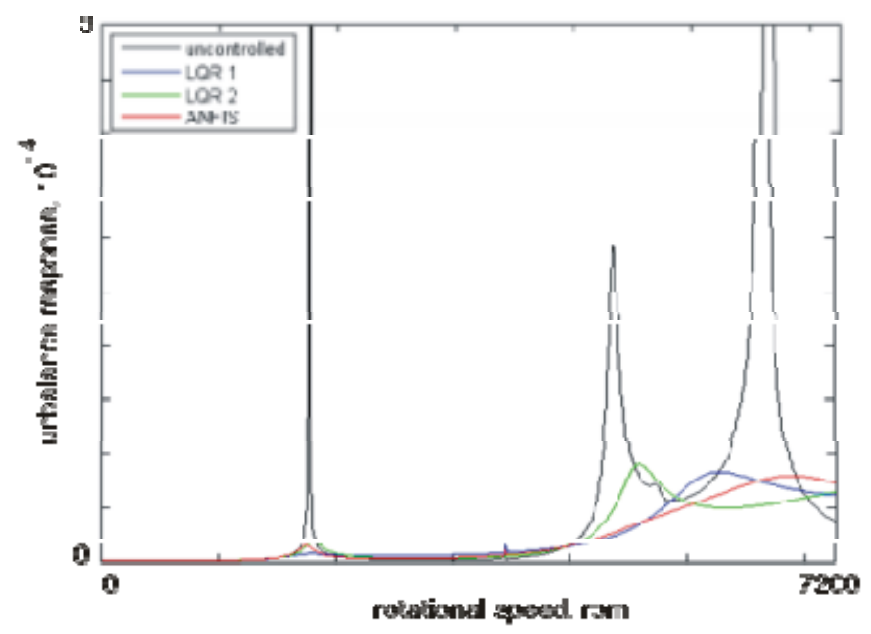

Figure 11. Response of the closed-loop system using tuned ANFIS controller compared to that of the system using LQR controller in the second scenario, evaluated at LPE bearing

\section{CONCLUSIONS}

The simulation shows that ANFIS controller works perfectly as approximator of the LQR controller. It also works well as a substitute of two different LQR controllers in which the first LQR controller is used for lower speeds whereas the second LQR controller for higher speeds. For this purpose, the ANFIS controller works quite well as a gain scheduler. Finally, it is shown that the ANFIS controller can also work as a better controller compared to LQR controller as it gives slightly better response at higher speeds. This is reached by inside-tuning the ANFIS controller.

\section{ACKNOWLEDGMENTS}

The authors extend their appreciation to the Deanship of Scientific Research and the Research Center of the College of Engineering at King Saud University for funding this work.

\section{References}

[1] Filbo, E.L.V.C., et al. A fuzzy logic controller for the active control of rotor vibration using an active magnetic bearing as an actuator. in ABCM Symposium Series in Mechatronics. 2012.

[2] Nagi, F.H., J.I. Inayat-Hussain, and S.K. Ahmed, Fuzzy bang-bang relay control of a singleaxis active magnetic bearing system. Simulation Modelling Practice and Theory, 2009. 17(10): p. 1734-1747.

[3] Chen, K.-Y., et al., A self-tuning fuzzy PID-type controller design for unbalance compensation in an active magnetic bearing. Expert Systems with Applications, 2009. 36(4): p. 8560-8570.

[4] Tung, P.-C., et al., Design of model-based unbalance compensator with fuzzy gain tuning mechanism for an active magnetic bearing system. Expert Systems with Applications, 2011. 38(10): p. 12861-12868.

[5] Couzon, P.Y. and J. Der Hagopian, Neuro-fuzzy Active Control of Rotor Suspended on Active Magnetic Bearing. Journal of Vibration and Control, 2007. 13(4): p. 365-384. 
[6] Gong, X. and D. Cao, Fuzzy proportional-integral-derivative control of an overhang rotor with double discs based on the active tilting pad journal bearing. Journal of Vibration and Control, 2012.

[7] Koroishi, E.H., V. Steffen, and J. Mahfoud, Fuzzy Control of Rotor System Using an Electromagnetic Actuator. MATEC Web of Conferences, 2012. 1: p. 09003.

[8] Borges, J.M., et al., Rotor-bearing vibration control system based on fuzzy controller and smart actuators. International Journal of Multiphysics, 2013. 7(3): p. 197-205.

[9] Li, W., P. Maißer, and H. Enge, Self-learning control applied to vibration control of a rotating spindle by piezopusher bearings. Proceedings of the Institution of Mechanical Engineers, Part I: Journal of Systems and Control Engineering, 2004: p. 218: 185.

[10] Lim, S., S.-M. Park, and K.-I. Kim, AI vibration control of high-speed rotor systems using electrorheological fluid. Journal of Sound and Vibration, 2005. 284(3-5): p. 685-703.

[11] Tammi, K., J. Hatonen, and S. Daley, Novel Adaptive Repetitive Algorithm for Active Vibration Control of a Variable-Speed Rotor. Journal of Mechanical Science and Technology, 2007. 21: p. 855-859.

[12] Tammi, K., Gradient-Based Repetitive Learning Control for Rotor Vibration Control. International Journal of Intelligent Control and Systems, 2008. 13: p. 222-232.

[13] Buttini, T.M. and R. Nicoletti, Self-Identification Algorithm for the Autonomous Control of Lateral Vibration in Flexible Rotors. International Journal of Rotating Machinery, 2012. 2012: p. 1-13.

[14] Rosyid, A., Vibration Intelligent Control of a Reduced-order Finite Element Model of a Rotor - Journal Bearing System. Mechanical Engineering Department, King Saud University, Riyadh, 2014. p. 9-54

[15] Jang, J.-S.R., ANFIS: Adaptive-Network-Based Fuzzy Inference System. IEEE Transactions on Systems, Man, and Cybernetics, 1993. 23(3).

[16] Jang, J.-S.R., C.-T. Sun, and E. Mizutani, ANFIS: Adaptive Neuro-Fuzzy Inference Systems, in Neuro-Fuzzy and Soft Computing: A Computational Approach to Learning and Machine Intelligence1997, Prentice Hall: New Jersey, USA. p. 335-363. 